\title{
The relationship between mortality and inflammatory markers and the systemic immune inflammatory index in patients in the intensive care unit with a pneumothorax as a complication of COVID-19 disease
}

\author{
Suleyman Anıl Akboga ${ }^{1}$ (1) - Anıl Gokce ${ }^{1} \cdot$ Merve Hatipoglu ${ }^{1} \cdot$ Muhammet Ali Beyoglu ${ }^{1} \cdot K_{\text {Kbilay Inan }}{ }^{1}$. \\ Aysegul Inci Sezen ${ }^{2} \cdot$ Hayriye Cankar Dal ${ }^{3} \cdot$ Yucel Akkas $^{1} \cdot$ Sema Turan $^{3} \cdot$ Bulent Kocer $^{1}$
}

Received: 8 June 2021 / Accepted: 4 August 2021 / Published online: 18 September 2021

(c) Royal Academy of Medicine in Ireland 2021

\begin{abstract}
Background A pneumothorax is common in patients admitted to the intensive care unit (ICU) with coronavirus disease (COVID-19) when non-invasive or invasive mechanical ventilation is performed to maintain adequate oxygenation. The aim of the present study was to investigate the effects of elevated inflammatory markers and an elevated systemic immune inflammatory index (SII) on mortality in this patient population.

Materials and methods Between March 2020 and May 2021, 124 patients with severe acute respiratory syndrome coronavirus 2 (SARS-CoV-2) polymerase chain reacion positviity who were admitted to the ICU in our hospital and diagnosed with and treated for a pneumothorax were evaluated retrospectively. Interleukin-6 (IL-6), C-reactive protein, neutrophil, lymphocyte, platelet and white blood cell levels were measured. These parameters were used to calculate the neutrophil-lymphocyte ratio (NLR) and SII, and the association of these parameters with pneumothorax-related mortality was examined.

Results This study included 39 female $(31.5 \%)$ and 85 male $(68.5 \%)$ patients. The mean age was $65.3 \pm 12.6$ years. Noninvasive mechanical ventilation was performed in $13(10.5 \%)$ patients, and $111(89.5 \%)$ patients received invasive mechanical ventilation. Tube thoracostomy was performed in 113 patients $(91.1 \%)$, and 11 patients (8.9\%) were treated with oxygen therapy. The factors affecting mortality in the pneumothorax patients were the Charlson Comorbidity Index (four or higher), IL-6 level and NLR. The IL-6 level was 53.4 in those who died versus 24.6 in those who survived $(p=0.017)$. The NLR in the patients who died was 16.9 as compared to 12.5 in those who survived $(p=0.011)$.

Conclusion Elevated markers of infection were associated with an increased risk of mortality in pneumothorax patients with COVID-19 who received invasive or non-invasive mechanical ventilation in the ICU. In this patient population, high levels of positive end-expiratory pressure should be avoided, and inflammatory marker levels and the SII should be closely monitored.
\end{abstract}

Keywords COVID-19 $\cdot$ Mortality $\cdot$ Pneumothorax $\cdot$ Prognostic factor

\section{Introduction}

Suleyman Anıl Akboga

doktor_anil_@hotmail.com

1 Thoracic Surgery Clinic, Ankara City Hospital, Çankaya, Turkey

2 Infectious Diseases and Clinical Microbiology Clinic, Istanbul Bakirkoy Dr. Sadi Konuk Training and Research Hospital, Bakırköy, Turkey

3 Intensive Care Clinic, Ankara City Hospital, Çankaya, Turkey
Coronavirus disease (COVID-19) can cause lung damage and insufficient respiratory capacity, both of which require non-invasive or invasive mechanical ventilation. The latter can lead to a pneumothorax when air fills the pleural space between the visceral pleura surrounding the lung parenchyma and the parietal pleura surrounding the chest wall, mediastinum and diaphragm. A spontaneous pneumothorax secondary to decreased lung compliance can also occur in COVID-19 disease cases. An iatrogenic pneumothorax can occur in patients with a positive severe acute respiratory 
syndrome coronavirus 2 (SARS-CoV-2) polymerase chain reaction (PCR) during hospitalization in the intensive care unit (ICU) due to the need for mechanical ventilation resulting from decreased respiratory capacity.

In retrospective studies of COVID-19 patients, a pneumothorax occurred in $1 \%$ of patients requiring hospitalization, $2 \%$ of patients admitted to the ICU and $1 \%$ of infected patients who died [1, 2]. Previous studies reported hyperinflammation in COVID-19 cases, together with an elevated Systemic Immune Inflammation Index (SII) and elevated neutrophil-lymphocyte ratio (NLR). However, the factors affecting mortality in COVID-19 patients remain unclear.

In the present study, we investigated the effects of an elevated SII and NLR on mortality in SARS-CoV-2 PCRpositive patients admitted to the ICU who developed a pneumothorax, with white blood cell (WBC), C-reactive protein (CRP) and interleukin-6 (IL-6) levels used to calculate the SII and NLR.

\section{Materials and methods}

Between March 2020 and May 2021, 124 patients admitted to the ICU in our hospital with SARS-CoV-2 PCR positivity and diagnosed with and treated for a pneumothorax were evaluated retrospectively. Between March 2020, the start of the pandemic in our hospital, and May 2021, 11,800 patients with COVID-19 disease were admitted to our ICU, of whom 3441 died. Thus, mortality due to COVID-19 was $29.16 \%$ in our ICU. Of the COVID-19 positive cases admitted to the ICU, 124 patients who had a pneumothorax and a positive PCR were included in the present study. Patients who had a high suspicion of COVID-19 infection and ground-glass opacity on computerized thorax tomography but without a positive PCR were not included. Furthermore, COVID-19 patients with a pneumothorax that developed outside the ICU and patients without SARS-CoV-2 PCR positivity were not included in the study.

The patients were divided into two groups: mild and severe. The severe group comprised patients with arterial blood gas levels of $\mathrm{PaO}_{2}<60 \mathrm{mmHg}$ or $\mathrm{PaCO}_{2}>50 \mathrm{mmHg}$ who were treated with invasive mechanical ventilation. The mild group comprised patients whose arterial blood gas results did not warrant invasive mechanical ventilation and who instead received non-invasive mechanical ventilation without intubation, with a normal oxygen mask or a high flow nasal cannula. Patients with a pneumothorax detected during follow-up were treated with a $28 \mathrm{~F}$ (French) chest tube. Those with a pneumothorax area of $<20 \%$ $(<2 \mathrm{~cm})$ according to the classification of the British Thorax Society pleural working group were medically treated and followed up with oxygenation without any intervention, under the condition that they had no symptoms and their general status was good [3]. IL-6, CRP, neutrophil, lymphocyte, platelet and WBC levels were measured in the patients who developed a pneumothorax when they were in the ICU. These parameters were used to calculate the NLR and SII. In eight patients, IL-6 levels were not measured. Thus, these patients were not included in the analyses (Fig. 1).

The SII was calculated as follows: (neutrophil count $\times$ platelet count)/lymphocyte count). The NLR was calculated as neutrophils/lymphocytes. The normal reference value ranges in our hospital for the inflammatory markers WBC, CRP, neutrophils, lymphocytes, platelets and IL-6 are $3.6-10.5 \times 10^{9} / \mathrm{L}, 0-5 \mathrm{~g} / \mathrm{L}, 1.5-7.7 \times 10^{9} / \mathrm{L}$, $1.1-4 \times 10^{9} / \mathrm{L}, 60-400 \times 10^{9} / \mathrm{L}$ and $0-3.4 \mathrm{pg} / \mathrm{mL}$, respectively. In addition, we used the modified Charlson Comorbidity Index (CCI) to calculate the comorbidity status of the patients at the time of inclusion in the study [4]. We examined the association of all the aforementioned parameters with pneumothorax-related mortality.

\section{Statistical analysis}

All statistical analyses were performed using IBM SPSS Statistics for Windows, version 22.0, released 2013 (IBM Corp., Armonk, NY). Numeric variables were analysed using the mean \pm standard deviation and median (min-max), and numbers and percentages were used for categorical variables. Prior to group comparisons of numeric variables, parametric test assumptions (normality and homogeneity of variances) were controlled. The differences between groups were analysed by a $T$ test in dependent groups. Categorical variables were analysed using Fisher's exact test. The Mann-Whitney $U$ test was used for the comparison of continuous variables. A $p$ value of $<0.05$ was considered statistically significant.

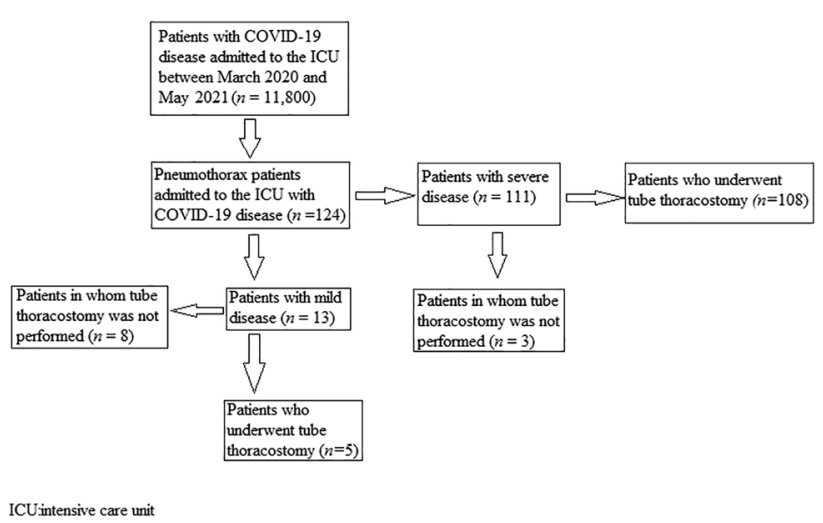

Fig. 1 Flow diagram of the patients included in the study 
Table 1 Demographic and clinical characteristics of the patients

\begin{tabular}{|c|c|c|c|}
\hline Variables & & $\begin{array}{l}N \text { (number of } \\
\text { patients) }\end{array}$ & Per cent \\
\hline \multirow[t]{2}{*}{ Gender } & Female & 39 & 31.5 \\
\hline & Male & 85 & 68.5 \\
\hline \multirow[t]{2}{*}{ Age } & $<65$ & 59 & 47.6 \\
\hline & $>65$ & 65 & 52.4 \\
\hline \multirow[t]{3}{*}{ Side } & Right hemithorax & 82 & 66.1 \\
\hline & Left hemithorax & 32 & 25.8 \\
\hline & Bilateral hemithorax & 10 & 8.1 \\
\hline \multirow{13}{*}{$\begin{array}{l}\text { Charlson } \\
\text { Comorbidity } \\
\text { Index }\end{array}$} & 0 & 5 & 4.0 \\
\hline & 1 & 3 & 2.4 \\
\hline & 2 & 14 & 11.3 \\
\hline & 3 & 12 & 9.7 \\
\hline & 4 & 24 & 19.4 \\
\hline & 5 & 24 & 19.4 \\
\hline & 6 & 14 & 11.3 \\
\hline & 7 & 13 & 10.5 \\
\hline & 8 & 9 & 7.3 \\
\hline & 9 & 2 & 1.6 \\
\hline & 10 & 1 & 0.8 \\
\hline & 12 & 2 & 1.6 \\
\hline & 14 & 1 & 0.8 \\
\hline \multirow[t]{2}{*}{ Prognosis } & Medium & 13 & 10.5 \\
\hline & Serious & 111 & 89.5 \\
\hline \multirow{2}{*}{$\begin{array}{l}\text { Treatment } \\
\text { applied to } \\
\text { pneumotho- } \\
\text { rax }\end{array}$} & Tube thoracostomy & 113 & 91.1 \\
\hline & Oxygen therapy & 11 & 8.9 \\
\hline
\end{tabular}

\section{Results}

The study included 39 female (31.5\%) and 85 male (68.5\%) patients. The mean age of the patients was $65.3 \pm 12.6$ years $(\min 23, \max 95)$. Fifty-nine $(47.6 \%)$ patients were aged $\leq 65$ years, and 65 patients $(52.4 \%)$ were older than 65 years. Eighty-one (65.3\%) of the 124 patients were smokers, with an average smoking history of $20.8 \pm 13.5$ packs/ year. Eighty-two (66.1\%) patients had right-sided pneumothorax, $32(25.8 \%)$ patients had left-sided pneumothorax, and $10(8.1 \%)$ patients had bilateral pneumothorax. Noninvasive mechanical ventilation was performed in $13(10.5 \%)$ patients, and invasive mechanical ventilation was performed in the other $111(89.5 \%)$ patients. Tube thoracostomy was performed in 113 (91.1\%) patients, and 11 (8.9\%) patients were treated with oxygen therapy. The mean hospitalization period was $23.7 \pm 17.2$ ( $\min 1$, $\max 120)$ days. Table 1 shows the clinical and demographic characteristics of the patients.

The median lymphocyte value was $51.2(\min 2.4$, max 2437). The median NLR was 16.6 ( $\min 0.06$, $\max 6284)$. The median CRP value was 103.9 ( $\min 2.4$, $\max 372.2$ ). The median IL-6 value was 46.7 ( $\min 4.8$, $\max 3802)$. The median SII value was 4234.7 .

When the factors affecting the prognosis were evaluated, no statistical difference was found in terms of the patient's sex, age or CCI. A high WBC count, pneumothorax in the right hemithorax and tube thoracostomy were associated with a worse prognosis. Table 2 shows the factors affecting the prognosis.
Table 2 Prognostic factors affecting the prognosis of the patient

\begin{tabular}{|c|c|c|c|c|c|c|}
\hline \multirow[t]{2}{*}{ Variables } & & \multicolumn{2}{|c|}{ Medium } & \multicolumn{2}{|c|}{ Serious } & \multirow[t]{2}{*}{$p$-value } \\
\hline & & $n$ & $\%$ & $n$ & $\%$ & \\
\hline \multirow[t]{2}{*}{ Gender } & Male & 11 & 84.6 & 74 & 66.7 & 0.187 \\
\hline & Female & 2 & 15.4 & 37 & 33.3 & \\
\hline \multirow[t]{2}{*}{ Age } & $<65$ & 8 & 61.5 & 51 & 45.9 & 0.284 \\
\hline & $>65$ & 5 & 38.5 & 60 & 54.1 & \\
\hline \multirow[t]{3}{*}{ Pneumothorax side } & Right hemithorax & 7 & 53.8 & 75 & 67.6 & 0.006 \\
\hline & Left hemithorax & 2 & 15.4 & 30 & 27 & \\
\hline & Bilateral hemithorax & 4 & 30.8 & 6 & 5.4 & \\
\hline \multirow[t]{2}{*}{ Tube thoracostomy } & No & 8 & 61.5 & 3 & 2.7 & $<0.001$ \\
\hline & Yes & 5 & 38.5 & 108 & 97.3 & \\
\hline \multirow[t]{2}{*}{ CCI } & $0-3$ & 3 & 23.1 & 31 & 27.9 & 0.711 \\
\hline & $>4$ & 10 & 76.9 & 80 & 72.1 & \\
\hline NLR & Median & 14.8 & & 16.2 & & 0.794 \\
\hline WBC & Median & 14.3 & & 10.8 & & 0.046 \\
\hline CRP & Median & 73.7 & & 104 & & 0.446 \\
\hline IL-6 & Median & 26.4 & & 49.4 & & 0.172 \\
\hline SII & Median & 3364 & & 4148 & & 0.893 \\
\hline
\end{tabular}

CCI Charlson Comorbidity Index, $N L R$ neutrophil-lymphocyte ratio, WBC white blood cell, CRPC-reactive protein, $I L-6$ interlockin-6, SII Systemic Immune Inflammation Index, StD standard deviation 
The factors affecting pneumothorax-related mortality were a CCI of 4 and above, elevated IL-6 level and elevated NLR. The NLR was 16.9 in the patients who died versus 12.5 in those who survived $(p=0.011)$. The IL-6 level was 53.4 in those who died as compared to 24.6 in the patients who survived $(p=0.017)$. Table 3 lists the prognostic factors affecting mortality.

\section{Discussion}

In a previous study, $1-2 \%$ of COVID-19 patients developed a pneumothorax [5]. In our study, between the pandemic period of March 2020 and May 2021, 11,800 patients with COVID-19 disease were admitted to the ICU in our hospital. In accordance with the literature, a pneumothorax developed in $124(1.05 \%)$ of these patients during the hospitalization period. Of these patients, 107 (86.3\%) died. Smoking is an important predisposing factor in the development of a pneumothorax [6]. In the present study, $81(65.3 \%)$ of the 124 pneumothorax patients were smokers. Seventy-eight (96.3\%) of these patients died, and $29(67.4 \%)$ of $43(34.7 \%)$ nonsmokers with a pneumothorax died $(p<0.001)$. Thus, in the present study, smoking was an important predisposing factor in the development of a pneumothorax, in accordance with the literature.

A pneumothorax may be secondary to a decrease in lung compliance in patients who have respiratory distress due to COVID-19 disease and who receive high positive end-expiratory pressure (PEEP) therapy, in conjunction with mechanical ventilation. COVID-19 disease is thought to induce cellular adhesion between type 1 and type 2 pneumocytes, leading to alveolar damage and a pneumothorax, depending on the inflammatory response of lung parenchyma and resulting ischemic damage [7]. In our study, we focused on patients with respiratory distress due to COVID-19 pneumonia who developed a pneumothorax during their time in the ICU. The pneumothorax occurred in the right hemithorax, left hemithorax and bilaterally in $82(66.1 \%), 32(25.8 \%)$ and $10(8.1 \%)$ patients, respectively. Mortality was $100 \%$ in the bilateral hemithorax cases. A pneumothorax may be more common in the right lung because the infection in COVID-19 disease spreads more easily from the right main bronchus, which is short and thick, to the right lung, leading to a decrease in lung compliance. In our study, 68 (82.9\%) of 82 patients who developed isolated right pneumothorax died, whereas 29 (90\%) of 32 patients who developed isolated left pneumothorax died. Thus, although a pneumothorax in the right
Table 3 Prognostic factors affecting mortality

\begin{tabular}{|c|c|c|c|c|c|c|}
\hline \multirow[t]{2}{*}{ Variables } & & \multicolumn{2}{|l|}{ No mortality } & \multicolumn{2}{|l|}{ Yes mortality } & \multirow[t]{2}{*}{$p$-value } \\
\hline & & $N$ (number of patients) & $\%$ & $N$ (number of patients) & $\%$ & \\
\hline \multirow[t]{2}{*}{ Gender } & Male & 14 & 82.4 & 71 & 66.4 & 0.187 \\
\hline & Female & 3 & 17.6 & 36 & 33.6 & \\
\hline \multirow[t]{2}{*}{ Age } & $<65$ & 11 & 64.7 & 48 & 44.9 & 0.13 \\
\hline & $>65$ & 6 & 35.3 & 59 & 55.1 & \\
\hline \multirow[t]{2}{*}{ Cigarette } & Yok & 14 & 82.4 & 29 & 27.1 & $<0.001$ \\
\hline & Var & 3 & 17.6 & 78 & 72.9 & \\
\hline Age (year) & Mean \pm StD & $59.5 \pm 17.2$ & & $66.2 \pm 11.6$ & & 0.142 \\
\hline \multirow[t]{3}{*}{ Pneumothorax side } & Right & 14 & 82.4 & 68 & 63.6 & 0.237 \\
\hline & Left & 3 & 17.6 & 29 & 27.1 & \\
\hline & Bilateral & 0 & 0 & 10 & 9.3 & \\
\hline \multirow[t]{2}{*}{ Tube thoracostomy } & No & 2 & 11.8 & 9 & 8.4 & 0.651 \\
\hline & Yes & 15 & 88.2 & 98 & 91.6 & \\
\hline \multirow[t]{2}{*}{ CCI } & $0-3$ & 9 & 52.9 & 25 & 23.4 & 0.011 \\
\hline & $>4$ & 8 & 47.1 & 82 & 76.6 & \\
\hline NLR & Median & 12.5 & & 16.9 & & 0.012 \\
\hline WBC & Median & 12.6 & & 11.0 & & 0.785 \\
\hline CRP & Median & 89.1 & & 106.7 & & 0.362 \\
\hline IL-6 & Median & 24.6 & & 53.4 & & 0.017 \\
\hline SII & Median & 3174.2 & & 4105.6 & & 0.100 \\
\hline \multirow[t]{2}{*}{ Prognosis } & Middle & 4 & 23.5 & 9 & 8.4 & 0.059 \\
\hline & Serious & 13 & 76.5 & 98 & 91.6 & \\
\hline
\end{tabular}

CCI Charlson Comorbidity Index, NLR neutrophil-lymphocyte ratio, WBC white blood cell, CRP C-reactive protein, $I L-6$ : interlockin-6, SII Systemic Immune Inflammation Index, StD standard deviation 
hemithorax was more common than in the left hemithorax, mortality was higher in the latter group. However, the difference was not statistically significant $(p=0.237)$. The prognosis in cases of a pneumothorax in the right hemithorax was poorer than that in cases of a pneumothorax in the left hemithorax and bilateral hemothorax, with a statistically significant difference $(p=0.006)$. Based on a review of the literature, there appear to be no data on the relationship of sides with COVID-19 disease and pneumothoraxes. Thus, our study can contribute to the literature in this area.

Previous studies reported that the development of a pneumothorax is an important prognostic factor in patients with COVID-19 disease [8-11]. In our study, 107 (86.3\%) of the 124 patients with a pneumothorax died. Based on these data and in accordance with the literature, a pneumothorax in patients with COVID-19 disease considerably increases the risk of mortality.

Yang et al. [12] and Zumla et al. [13] found that the inflammatory response is an important prognostic factor in COVID-19 disease and that the occurrence of an inflammatory cytokine storm increases mortality in patients with the disease. Wan et al. [14] stated that a cytokine storm in patients with COVID-19 disease increased morbidity, including severe complications, which increased the risk of mortality in this patient population. In accordance with the literature and based on our findings, we conclude that an increase in IL-6 levels increased pneumothorax-related mortality in patients with COVID-19 disease.

In a meta-analysis that included three studies on patients with COVID-19 disease and a poor prognosis, the authors reported a statistically significant increase in WBC and IL-6 levels in COVID-19 patients who died [15-17]. In our study, similar to the literature, although a high WBC count in the early period had a significant effect on the prognosis $(p=0.0046)$, we detected no statistically significant relationship between CRP and WBC levels and mortality.

In previous studies, COVID-19 patients with a poor prognosis had lymphocytopenia affecting CD4+ and CD8 + cells, as well as an increase in the NLR due to a decrease in monocytes and eosinophils and an increase in neutrophils $[18,19]$. In our study, pneumothorax patients with a high NLR had a higher mortality rate than those with a lower NLR. This finding was statistically significant $(p=0.012)$ and consistent with the literature.

In a study on COVID-19 patients in Italy, Fois et al. [20] reported decreased survival among patients with a high SII. The SII is used as a prognostic marker in patients with sepsis [21]. SII has also been shown to be associated with worse survival in small cell lung cancer, hepatocellular carcinoma, head and neck cancers, colorectal cancer and gastric cancer [22-26]. In the present study, there was no statistical association between the SII and prognosis and mortality in
COVID-19 patients with a pneumothorax ( $p=0.893$ and $p=0.100$, respectively).

Recent studies reported the presence of SARS-CoV-2 viral RNA in pleural fluid samples taken from a chest drain $[27,28]$. To prevent such occurrences, the team that inserts the chest tube and the team that manages the drain after chest tube insertion should carefully apply COVID-19 protective measures.

\section{Limitations of the study}

The main limitations of this study were its retrospective nature and the small number of patients. In addition, this was a single centre rather than a multi-centre study, the patients were evaluated by more than one physician, it was not compared with other pneumothorax patients, and the duration of the study was short.

\section{Conclusions}

A pneumothorax is common during non-invasive and invasive mechanical ventilation performed for adequate oxygenation in COVID-19 patients admitted to the ICU with pneumonia. Pneumothorax-related mortality in this patient population increases in accordance with the presence of a cytokine storm (i.e. increased levels of inflammatory markers), an elevated CII and an increased NLR. In patients with COVID-19 disease who receive invasive or non-invasive mechanic ventilation, high PEEP levels should be avoided, and inflammatory marker levels and the SII should be closely monitored.

\section{Declarations}

Conflict of interest The authors declare that they have no conflict of interest.

Ethical approval This study was approved by the ethics committee of Ankara City Hospital, Turkey. Date: 02/06/2021/No: E2-21-572.

\section{References}

1. Chen N, Zhou M, Dong X et al (2020) Epidemiological and clinical characteristics of 99 cases of 2019 novel coronavirus pneumonia in Wuhan, China: a descriptive study. Lancet 395:507-513

2. Yang F, Shi S, Zhu J et al (2020) Analysis of 92 deceased patients with COVID-19. J Med Virol. https://doi.org/10.1002/jmv.25972

3. Henry M, Arnold T, Harvey J, BTS pleural group (2003) BTS guidelines for the management of spontaneous pneumothorax. Thorax 58(Suppl II):39-52 
4. Beddhu S, Bruns FJ, Saul M et al (2000) A simple comorbidity scale predicts clinical outcomes and costs in dialysis patients. Am J Med 108(8):609-613

5. Yang X (2020) Clinical course and outcomes of critically ill patients with SARS-CoV-2 pneumonia in Wuhan, China: a singlecentered, retrospective, observational study. Lancet Respir Med $8: 475-481$

6. Gonca E, Chousein U, Çörtük M et al (2020) Is there any effect of smoking status on severity and mortality of hospitalized patients with COVID-19 pneumonia? Tuberk Toraks 68(4):371-378. https://doi.org/10.5578/tt.70352

7. Liu K, Zeng Y, Xie P et al (2020) COVID-19 with cystic features on computed tomography: a case report. Medicine (Baltim) 99(18):e20175

8. McGuinness G, Zhan C, Rosenberg N et al (2020) High incidence of barotrauma in patients with COVID-19 infection on invasive mechanical ventilation. Radiology. https://doi.org/10.1148/radiol. 2020202352

9. López Vega JM, Parra Gordo ML, DiezTascón A et al (2020) Pneumomediastinum and spontaneous pneumothorax as an extrapulmonary complication of COVID-19 disease. Emerg Radiol. https://doi.org/10.1007/s10140-020-01806-0

10. Sihoe ADL, Wong RHL, Lee ATH et al (2004) Severe acute respiratory syndrome complicated by spontaneous pneumothorax. Chest 125:2345-2351

11. Das KM, Lee EY, Al Jawder SE et al (2015) Acute Middle East respiratory syndrome coronavirus: temporal lung changes observed on the chest radiographs of 55 patients. AJR Am J Roentgenol 205:W267-S274

12. Yang PH, Ding YB, Xu Z et al (2020) Epidemiological and clinical features of COVID-19 patients with and without pneumonia in Beijing, China. MedRxiv. https://doi.org/10.1101/2020.02.28. 20028068

13. Zumla A, Hui DS, Azhar EI et al (2020) Reducing mortality from 2019-nCoV: host-directed therapies should be an option. Lancet 395:e35-36. https://doi.org/10.1016/S0140-6736(20)30305-6

14. Wan SX, Yi QJ, Fan SB et al (2020) Characteristics of lymphocyte subsets and cytokines in peripheral blood of 123 hospitalized patients with 2019 novel coronavirus pneumonia (NCP). MedRxiv. https://doi.org/10.1101/2020.02.10.20021832

15. Yang X, Yu Y, Xu J et al (2020) Clinical course and outcomes of critically ill patients with SARS-CoV-2 pneumonia in Wuhan, China: a single-centered, retrospective, observational study. Lancet Respir Med 8(5):475-481

16. Ruan Q, Yang K, Wang W et al (2020) Clinical predictors of mortality due to COVID-19 based on an analysis of data of 150 patients from Wuhan, China. Intensive Care Med 46(5):846-848

17. Zhou F, Yu T, Du R et al (2020) Clinical course and risk factors for mortality of adult inpatients with COVID-19 in Wuhan,
China: a retrospective cohort study. Lancet (Lond, Engl) 395(10229): 1054-1062

18. Zheng Y, Xu H, Yang M et al (2020) Epidemiological characteristics and clinical features of 32 critical and 67 noncritical cases of COVID-19 in Chengdu. J Clin Virol 127:104366

19. Henry BM, Oliveira MHS, de Benoit S et al (2020) Hematologic, biochemical and immune biomarker abnormalities associated with severe illness and mortality in coronavirus disease 2019 (COVID-19): a meta-analysis. Clin Chem Lab Med. https://doi. org/10.1515/cclm-2020-0369

20. Alessandro GF, Panagiotis P, Valentino S et al (2020) The systemic inflammation index on admission predicts in-hospital mortality in COVID-19 patients. Molecules 20(23):5725. https://doi. org/10.3390/molecules25235725

21. Lagunas-Alvarado M, Mijangos-Huesca FJ, Terán-González JO et al (2017) Systemic immune inflammatory index in sepsis. Med Int Méx 33:303-309

22. Hong X, Cui B, Wang M et al (2015) Systemic immune-inflammation index, based on platelet counts and neutrophil-lymphocyte ratio, is useful for predicting prognosis in small cell lung cancer. Tohoku J Exp Med 236:297-304. https://doi.org/10.1620/tjem. 236.297

23. Hu B, Yang X-R, Xu Y et al (2014) Systemic immune-inflammation index predicts prognosis of patients after curative resection for hepatocellular carcinoma. Clin Cancer Res 20:6212-6222. https://doi.org/10.1158/1078-0432.CCR-14-0442

24 Dong M, Shi Y, Yang J et al (2020) Prognostic and clinicopathological significance of systemic immune-inflammation index in colorectal cancer: a meta-analysis. Ther Adv Med Oncol. https:// doi.org/10.1177/1758835920937425

25. Hirahara N, Matsubara T, Fujii Y et al (2020) Comparison of the prognostic value of immunoinflammation-based biomarkers in patients with gastric cancer. Oncotarget 11:2625-2635. https:// doi.org/10.18632/oncotarget.27653

26. Akkas EA, Yucel B (2021) Prognostic value of systemic immune inflammation index in patients with laryngeal cancer. Eur Arch Otorhinolaryngol 278:1945-55

27. Pieracci FM, Burlew CC, Spain D et al (2020) Tube thoracostomy during the COVID-19 pandemic: guidance and recommendations from the AAST acute care surgery and critical care committees. Trauma Surg Acute Care Open 5:e000498

28. Schaller T, Hirschbühl K, Burkhardt K et al (2020) Postmortem examination of patients with COVID-19. JAMA 323:2518-2520

Publisher's note Springer Nature remains neutral with regard to jurisdictional claims in published maps and institutional affiliations. 